\title{
Quantum Computing with Trapped Ion Hyperfine Qubits
}

\author{
B. B. Blinov, ${ }^{1,3}$ D. Leibfried, ${ }^{2}$ C. Monroe, ${ }^{1}$ and D. J. Wineland ${ }^{2}$
}

Received January 30, 2004; accepted March 5, 2004

We discuss the basic aspects of quantum information processing with trapped ions, including the principles of ion trapping, preparation and detection of hyperfine qubits, single-qubit operations and multi-qubit entanglement protocols. Recent experimental advances and future research directions are outlined.

KEY WORDS: Ion trapping; hyperfine qubits; quantum gates; qubit detection; qubit initialization; entanglement.

PACS: $03.67 . \mathrm{Lx}, 32.80 . \mathrm{Pj}, 32.80 . \mathrm{Qk}, 42.50 . \mathrm{Vk}$.

\section{OVERVIEW}

Trapped atomic ions were first proposed as a viable quantum computing candidate by Cirac and Zoller in 1995. ${ }^{(1)}$ Almost 10 years later, the trapped ion system remains one of the few candidates that satisfies the general requirements for a quantum computer as outlined by DiVincenzo $^{(2)}$ : (i) a scalable system of well-defined qubits, (ii) a method to reliably initialize the quantum system, (iii) long coherence times, (iv) existence of universal gates, and (v) an efficient measurement scheme. Most of these requirements have been demonstrated experimentally with trapped ions, and there exist straightforward (albeit technically difficult) paths to solving the remaining problems.

Experimental approaches in ion trap quantum computing can be divided by the type of qubit, in terms of the qubit level energy splitting,

\footnotetext{
${ }^{1}$ FOCUS Center and Department of Physics, University of Michigan Ann Arbor, MI 48109, USA; e-mail: bblinov@umich.edu

${ }^{2}$ Time and Frequency Division, National Institute of Standards and Technology Boulder, CO 80303, USA.

${ }^{3}$ To whom correspondence should be addressed.
} 
and the couplings required to drive quantum logic gates between the qubit states. The two primary types of trapped ion qubit architectures are optical qubits derived from a ground state and an excited metastable state separated by an optical frequency, and hyperfine qubits derived from electronic ground-state hyperfine (HF) levels separated by a microwave frequency. In this paper, we highlight the latter case of HF qubits. For a review of optical qubits we refer to the paper by R. Blatt included in this volume. More general accounts and various reviews are given in Refs. 3-16. Moreover, the Advanced Research and Development Activity (ARDA) has posted and updated a "roadmap" to highlight accomplishments and problems with the various possible implementations of quantum information processing including those based on ion traps; various references can be found at this site (http://qist.lanl.gov). Many experimental groups worldwide have addressed various aspects of trapped-ion quantum information processing; these include groups at University of Aarhus, IBM-Almaden, NIST-Boulder, University of Hamburg, McMaster University, University of Innsbruck, Los Alamos, University of Michigan, Max Planck Inst.Garching, Oxford University, and NPL-Teddington.

\section{ION TRAPS}

Ion traps come in various forms ${ }^{(17)}$; for brevity, we restrict our discussion to the linear RF (Paul) trap shown schematically in Fig. 1. Linear Paul traps with particular application to quantum information processing are discussed by various groups; see, for example, Refs. 3-16 and 1827. The linear trap is essentially a quadrupole mass filter plugged on the axis by superimposing a static electric potential well. In the $x, y$ plane of the figure, ions are bound by a ponderomotive pseudopotential

$$
U_{x, y}(\mathbf{r})=\frac{q^{2}}{2 m \Omega_{T}^{2}}\left\langle E^{2}(\mathbf{r})\right\rangle \simeq \frac{q^{2} V_{0}^{2}}{4 m \Omega_{T}^{2} R^{4}}\left(x^{2}+y^{2}\right),
$$

where $q$ is the ion's charge, $m$ its mass, $E$ is the RF electric field (resulting from a potential $V_{0} \cos \left(\Omega_{T} t\right)$ applied to the dark electrodes of Fig. 1), $\mathbf{r}$ is the radial distance from the trap axis, and $R$ is the distance between the trap axis and the nearest electrode surface. The oscillation frequency of an ion in this pseudopotential is given by

$$
\omega_{x, y} \simeq \frac{q V_{0}}{\sqrt{2} \Omega_{T} m R^{2}},
$$




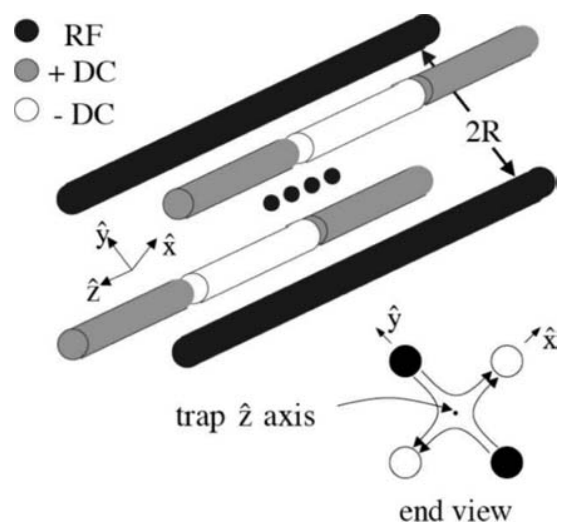

Fig. 1. Electrode configuration for a linear RF (Paul) trap. A common RF potential $V_{0} \cos \left(\Omega_{T} t\right)$ is applied to the dark electrodes; the other electrodes are held at RF ground through capacitors (not shown) connected to ground. The lower-right portion of the figure shows the $x, y$ electric fields from the applied RF potential at an instant when the RF potential is positive relative to ground. A static electric potential well is created (for positive ions) along the $z$-axis by applying a positive potential to the outer segments (grey) relative to the center segments (white).

where we assume the pseudopotential approximation $\left(\omega_{x, y} \ll \Omega_{T}\right)$ and assume that $\omega_{z} \ll \omega_{x, y}$ so that the static radial forces are much smaller than the pseudopotential forces. From the form of Eq. (1), we see that the ions seek the region of minimum $|E(\mathbf{r})|$. As an example, for some typical experiments using ${ }^{9} \mathrm{Be}^{+}$ions, ${ }^{(28)} q=1 e, V_{0}=500 \mathrm{~V}, \Omega_{T} / 2 \pi \simeq 100 \mathrm{MHz}$, and $R=200 \mu \mathrm{m}$, so that $\omega_{x, y} / 2 \pi \simeq 24 \mathrm{MHz}$.

For the purposes of quantum computing, to a good approximation, we can view the linear trap as providing a three-dimensional harmonic well for ion qubits, where the strength of the well in two directions ( $x$ and $y$ in Fig. 1) is much stronger than in the third direction $(z)$. When a small number of ions is trapped and cooled, each ion seeks the bottom of the trap well, but the mutual Coulomb repulsion between ions results in an equilibrium configuration in the form of a linear array, like beads on a string. To give an idea of array size, two ions in such a trap are spaced by $2^{1 / 3} s$, and three ions are spaced by $(5 / 4)^{1 / 3} s$ where $s \equiv q^{2} /\left(4 \pi \epsilon_{\mathrm{o}} m \omega_{z}^{2}\right)^{1 / 3}$. Expressed equivalently, for singly charged ions, the spacing parameter in micrometers is $s(\mu \mathrm{m})=15.2\left(M(u) v_{z}^{2}(\mathrm{MHz})\right)^{-1 / 3}$, where the ion's mass is expressed in a.m.u. and the axial $z$ frequency in $\mathrm{MHz}$. For $v_{z}=5 \mathrm{MHz}$, two ${ }^{9} \mathrm{Be}^{+}$ions are separated by $3.15 \mu \mathrm{m}$.

Although simple gate operations among a few ion qubits have been demonstrated, a viable quantum computer architecture must accomodate 
very large number of qubits. As the number of ions in a trap increases, several difficulties are encountered. For example, the addition of each ion adds three vibrational modes. It soon becomes nearly impossible to spectrally select the desired vibrational mode unless the speed of operations is slowed to undesirable levels. ${ }^{(4,29)}$ Furthermore, since error correction will most likely be incorporated into any large processor, it will be desirable to reset (or possibly measure) ancilla qubits without disturbing the coherence of logical qubits. Since ion qubits are typically reset by means of statedependent laser scattering, the scattered light from ancilla qubits held in a common trap may disturb the coherence of the logical qubits.

For these and other reasons, it appears that a scalable ion-trap system must incorporate arrays of interconnected traps, each holding a small number of ions. The information carriers between traps might be photons, ${ }^{(19,30-32)}$ or ions that are moved between traps in the array. In the latter case, a "head" ion held in a movable trap could carry the information by moving from site-to-site as in the proposal of Ref. 22. Similarly, as suggested in Refs. 4 and 26, qubit ions themselves could be shuttled around in an array of interconnected traps. In this scheme, the idea is to move ions between nodes in the array by applying time-dependent potentials to "control" electrode segments. To perform logic operations between selected ions, these ions are transferred into an "accumulator" trap for the gate operation. Before the gate operation is performed, it may be necessary to sympathetically re-cool the qubit ions with "refrigerant" ions. ${ }^{(4,26,33-35)}$ Subsequently, these ions are moved to memory locations or other accumulators. This strategy always maintains a relatively small number of motional modes that must be considered and minimizes the problems of ion-laser-beam addressing using focused laser beams. Such arrays also enable highly parallel processing and ancilla qubit readout in a separate trapping region so that the logical ions are shielded from the scattered laser light.

Most gate schemes for trapped ions have a speed that is limited to, or proportional to, the oscillation frequency of the ions in the trap. From Eq. 2, we therefore want to maximize $V_{0} / R^{2}$. As $R$ becomes smaller it is more difficult to control the relative dimensions of the electrode structures. Refs. 3-16 and 18-27 discuss some approaches to making small traps with accurate dimensions.

\section{TRAPPED ION HYPERFINE QUBITS}

Ions can be confined for days in an ultra-high vacuum with minimal perturbations to their internal atomic structure, making particular internal 
states ideal for representing quantum bits. Electric field perturbations are small, because localized ions experience a vanishing time-averaged electric field. Although magnetic field perturbations to internal structure can be important, the coherence between two internal levels can be made magnetic field-insensitive (to lowest order) by operating near an extremum of the energy separation between the two levels with respect to the magnetic field. Qubit coherence in such atomic ground states has been observed for times exceeding $10 \mathrm{~min}$ in the context of trapped ion frequency standards. ${ }^{(36,37)}$

Qubits stored in metastable levels separated by optical frequencies $(1,38)$ enjoy the simplicity of single-photon optical transitions, provided the radiative decay rate is sufficiently slow (some weakly allowed optical transitions in atomic ions have lifetimes $\gg 1 \mathrm{~s}$ ) However, phase-stable narrowlinewidth lasers are required in order to realize the full benefit of the long decay times. ${ }^{(39,40)}$ In addition, Stark shifts from coupling to non-resonant allowed transitions become important for these longer qubit lifetimes since the laser intensity must be high for appreciable transition rates. ${ }^{(41)}$

Ground state hyperfine levels, or states of nuclear vs. electronic spin, typically separated by microwave frequencies, have extremely long radiative lifetimes. Trapped ion HF levels are arguably the most attractive choice for qubit states, and form the thesis of this paper. Figure 2 displays the lowest energy levels of the ${ }^{111} \mathrm{Cd}^{+}$ion, for concreteness (nuclear spin $I=1 / 2$ ). We will be interested primarily in two electronic states, the ${ }^{2} S_{1 / 2}\left(F=1, m_{F}=\right.$ $0)$ and ${ }^{2} S_{1 / 2}\left(F=0, m_{F}=0\right)$ hyperfine ground states (denoted by $|\downarrow\rangle$ and $|\uparrow\rangle$ respectively), separated by frequency $\omega_{\mathrm{HF}}\left(\omega_{\mathrm{HF}} / 2 \pi \simeq 14.53 \mathrm{GHz}\right.$ for ${ }^{111}\left(\mathrm{Cd}^{+}\right)$. These long-lived spin states will form the basis for a quantum bit. Other candidate ions with similar HF structure (non-zero nuclear spin) include ${ }^{9} \mathrm{Be}^{+},{ }^{25} \mathrm{Mg}^{+},{ }^{43} \mathrm{Ca}^{+},{ }^{87} \mathrm{Sr}^{+},{ }^{137} \mathrm{Ba}^{+},{ }^{173} \mathrm{Yb}^{+}$, and ${ }^{199} \mathrm{Hg}^{+}$.

\subsection{Qubit Initialization and Detection}

Standard optical pumping techniques allow the HF qubits to be initialized into either $|\downarrow\rangle$ or $|\uparrow\rangle$ states. Subsequent detection of the spin states can be accomplished using the technique of quantum jumps. ${ }^{(42)}$ For example, in ${ }^{111} \mathrm{Cd}^{+}$, a circularly polarized laser beam resonant with the ${ }^{2} S_{1 / 2}-{ }^{2} P_{3 / 2}$ transition near $\lambda \simeq 214.5 \mathrm{~nm}$ (Fig. 2) scatters many photons if the atom is in the $|\downarrow\rangle$ spin state (a "cycling" transition), but essentially no photons when the atom is in the $|\uparrow\rangle$ spin state. Even if a modest number of the scattered photons are detected, the efficiency of discrimination between these two states approaches $100 \%$. In general, the HF qubit detection efficiency with such cycling transition is given by 


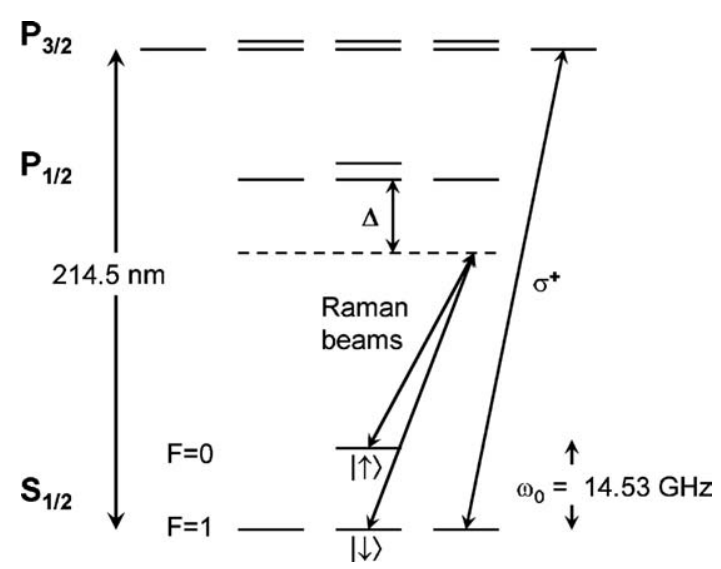

Fig. 2. Electronic (internal) energy levels (not to scale) of the ${ }^{111} \mathrm{Cd}^{+}$ion. The ${ }^{2} S_{1 / 2}\left(F=1, m_{F}=0\right)$ and ${ }^{2} S_{1 / 2}\left(F=0, m_{F}=0\right)$ hyperfine ground states (denoted by $|\downarrow\rangle$ and $|\uparrow\rangle$ respectively), separated in frequency by $\omega_{H F} / 2 \pi \simeq 14.53 \mathrm{GHz}$, and magnetic field insensitive to first order, form the basis of a quantum bit. Detection of the internal HF state is accomplished by illuminating the ion with a $\sigma^{+}$-polarized "detection" beam near $\lambda_{C d} \simeq 214.5 \mathrm{~nm}$ and observing the fluorescence from the cycling transitions between $|\downarrow\rangle$ and the ${ }^{2} P_{3 / 2}\left|\left(F=2, m_{F}=2\right)\right\rangle$ state. The excited $\mathrm{P}$ state has radiative linewidth $\gamma_{e} / 2 \pi \simeq 47 \mathrm{MHz}$. Also drawn are a pair of $\sigma^{+}$-polarized Raman beams that are used for quantum logic gates.

$1-\left(M / \epsilon_{\text {phot }}\right)\left(\gamma_{e} / \omega_{\mathrm{HF}}\right)^{2}$, where $M$ includes appropriate atomic branching ratios and is of order unity, and $\epsilon_{\text {phot }}$ is the photon detection efficiency of the ion fluorescence. In Fig. 3, the number of photons scattered in $0.2 \mathrm{~ms}$ by a single ${ }^{111} \mathrm{Cd}^{+}$ion (net quantum-efficiency $\epsilon_{\text {phot }} \sim 10^{-3}$ ) is plotted for the "bright" $(|\downarrow\rangle)$ and the "dark" $(|\uparrow\rangle)$ states. By placing the discriminator between two and three detected photons, a HF qubit detection efficiency of $99.7 \%$ is realized.

\subsection{HF Qubit Rotations: Single Qubit Gates}

Single-qubit rotations of HF states can be accomplished by either applying microwave radiation tuned to the energy splitting between the two levels $\omega_{\mathrm{HF}}$, or by driving stimulated Raman transitions (SRT) with two laser fields that are properly detuned from an excited state and differ in frequency by $\omega_{\mathrm{HF}}$.

When microwaves are used, it is necessary to efficiently couple radiation with $\sim \mathrm{cm}$ wavelengths into a sub-millimeter size ion trap. Low gain microwave horns (with small opening angles) provide reasonable means of generating a strong beam of microwaves. Rabi frequencies approaching 100 $\mathrm{kHz}$ have been achieved with modest microwave powers $(<1 \mathrm{~W})$ applied 


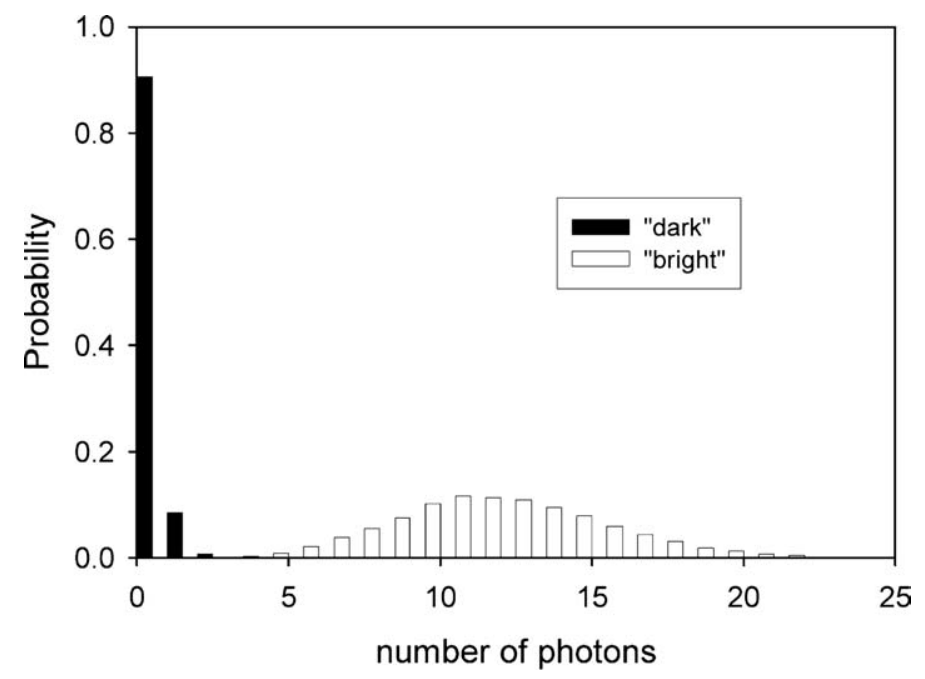

Fig. 3. Detection histograms of a single trapped ${ }^{111} \mathrm{Cd}^{+}$ion. The white bars correspond to the distribution of the number of fluorescence photons detected by a CCD camera for a ${ }^{111} \mathrm{Cd}^{+}$ion prepared initially in the $|\downarrow\rangle$ state upon application of a $\sigma^{+}$-polarized detection laser for $0.2 \mathrm{~ms}$. The black bars correspond to the photon distribution for the ion initially prepared in the $|\uparrow\rangle$ state under the same conditions. The very small overlap between the two distributions corresponds to a detection efficiency of $>99.7 \%$.

through a horn within $10 \mathrm{~cm}$ of the ion. Microwave qubit rotations can easily be made very clean by using stable RF sources, and are useful for joint rotations of all qubits. However, individual addressing of trapped ions with microwaves is difficult, unless magnetically sensitive qubit states are employed and substantial magnetic field gradients are applied. ${ }^{(44)}$

In the case of ${ }^{2} S_{1 / 2}$ HF qubit rotations with SRT, ${ }^{(5)}$ two co-propagating laser fields are applied to the ion, each with a detuning $\Delta \gg \gamma_{e}$ from an excited ${ }^{2} P_{1 / 2}$ or ${ }^{2} P_{3 / 2}$ state, denoted by $|e\rangle$ with radiative linewidth $\gamma_{e}$. The difference frequency of the two fields is set to the HF qubit resonance, resulting in an effective field that coherently rotates the HF qubit similar to the microwave case, except because the SRT laser beams can be focussed, individual ions can be addressed. The SRT Rabi frequency is given by $\Omega_{\mathrm{SRT}}=g_{1} g_{2}^{*} / \Delta$, where $g_{i}$ are the resonant Rabi frequencies for the two laser beams respectively driving transitions to the excited state $|e\rangle$. The probability of spontaneous emission from off-resonant excitation to the state $|e\rangle$ during a SRT $\pi$-pulse decreases with increasing $\Delta$ as $P_{s e} \simeq \gamma_{e} / \Delta$.

However, $\Delta$ cannot be increased indefinitely. In order for SRT to effectivly couple HF states and effectively flip the state of electron + 
nuclear spin, the excited state $|e\rangle$ must be populated for a sufficient time so that the spin-orbit interaction allows the spin to flip. In the case of $S_{1 / 2}$ hyperfine ground state qubits coupled to excited atomic $P_{1 / 2}$ or ${ }^{2} P_{3 / 2}$ states, this time-scale is set by the inverse fine-structure splitting $1 / \Delta_{\mathrm{FS}}$, and we find that the probability of spontaneous emission in a given qubitdependent operation is roughly $P_{s e} \simeq \gamma_{e} / \Delta_{\mathrm{FS}}$, which can be as low as $10^{-5}$ in candidate ion species. ${ }^{(13)}$

\subsection{Interactions Between HF Qubits: Entangling Qubit Gates}

Trapped ion qubits can be controllably coupled through their mutual Coulomb interaction. While the internal HF qubit states are essentially unaffected by the Coulomb interaction directly, external control fields can generate an effective coupling between qubits that relies on a qubit statedependent force. This external control field can thus entangle trapped ion qubits through the "data bus" represented by the Coulomb interaction.

For a qubit stored in atomic $S_{1 / 2} \mathrm{HF}$ ground states $|\downarrow\rangle$ and $|\uparrow\rangle$, the coupling to an ion's position $\hat{\mathbf{x}}$ proceeds via a dipole coupling of one (or both) qubit states to an excited atomic $P_{1 / 2}$ or $P_{3 / 2}$ state $|e\rangle$ having the form

$$
H_{I}=-\left(\hat{\mu}_{\uparrow, \mathbf{e}}+\hat{\mu}_{\downarrow, \mathbf{e}}\right) \cdot \mathbf{E}(\hat{\mathbf{x}}) .
$$

Here, $\hat{\mu}_{\mathbf{S}, \mathbf{e}}$ is the electric dipole operator between qubit state $|S\rangle$ and $|e\rangle$, and $\mathbf{E}(\hat{\mathbf{x}})$ is the electric field of the laser as a function of the position of the ion $\hat{\mathbf{x}}$. This interaction can be sequentially or simultaneously applied to different trapped ion qubits in order to generate entanglement. While the ion position is thus used to entangle trapped ion qubits, successful gate schemes rely on the quantum state of position not becoming ultimately entangled with the qubit states following the gate. Below we discuss two of the most common methods for coupling the qubit and position of trapped ions: motion-sensitive stimulated Raman transitions, and qubit state-dependent forces.

\subsubsection{Motion-sensitive stimulated Raman transitions}

Optical stimulated Raman transitions are not only useful for simple HF qubit rotation operations as discussed above, but can be critical for coupling qubit states to the external motional state of the ions. In this case, the two Raman beams are directed onto the ion(s) with a nonzero wavevector difference $\delta \mathbf{k}$ along the relevant direction of motion to be coupled. For a single ion, the resulting coupling under the rotating wave 
approximation $^{(4)}$ is

$$
H=\eta \Omega_{\mathrm{SRT}}\left(\sigma_{+} \mathrm{e}^{\mathrm{i} \eta\left(a+a^{\dagger}\right)}+\sigma_{-} \mathrm{e}^{\mathrm{i} \eta\left(a+a^{\dagger}\right)}\right),
$$

where $\eta=\delta \mathbf{k} \cdot \mathbf{x}_{\mathbf{0}}$ is the Lamb-Dicke parameter associated with the coordinate $x$ and $x_{0}=(\hbar / 2 m \omega)^{1 / 2}$ is the 0 -point spread of the ion wavepacket. The raising and lowering operators for the qubit (motion) are given by $\sigma_{+}$ and $\sigma_{-}\left(a^{\dagger}\right.$ and $\left.a\right)$. The Lamb-Dicke parameter $\eta$ sets the scale for the coupling between the light field and the position of the ion, related to the gradient of the light field experienced by the ion.

We provide two specific examples of SRT couplings from Eq. 4 that can be exploited for entangling the quantum state of a single ion with a collective mode of motion. Subsequently, similar operations can be applied to other ions sharing the motion, resulting in a net entangling quantum gate between ion qubits. In the original Cirac-Zoller proposal, ${ }^{(1)}$ the difference frequency between the two stimulated Raman fields is tuned to a "motional sideband" at frequency $\omega_{\mathrm{HF}} \pm k \omega$, where $k$ is an integer describing the sideband order. Raman sideband operations coherently rotate the $\mathrm{HF}$ qubit state while simultaneously affecting the quantum state of motion. The resulting coupling for the first lower sideband $(k=-1)$ takes the form of the classic Jaynes-Cummings Hamiltonian:

$$
H_{-1}=\eta \Omega_{\mathrm{SRT}}\left(\sigma^{+} a+\sigma^{-} a \dagger\right) .
$$

The above expression assumes that the ion is confined to within the Lamb-Dicke limit, although this is not essential. For a single trapped ion initially prepared in the vibrational ground state $(|0\rangle)$, this coupling results in the mapping of an arbitrary qubit state $(\alpha|\downarrow\rangle+\beta|\uparrow\rangle)|0\rangle$ to $|\downarrow\rangle(\alpha|0\rangle+$ $\beta|1\rangle)$. This interaction is the basis for the Cirac-Zoller ${ }^{(1)}$ and MølmerSørensen ${ }^{(46,47)}$ quantum logic gate schemes.

When a pair of non-copropagating Raman beams are tuned to the carrier transition, multi-qubit entangling gates can also be realized. In this case, we find that qubit transitions are driven without accompanying motional state transitions, although the qubit Rabi frequency acquires a dependence upon the motional state of the form

$$
\Omega_{n, n}=\eta \Omega_{\mathrm{SRT}} e^{-\eta^{2} / 2} L_{n}\left(\eta^{2}\right)
$$

where $L_{n}(X)$ is the Laguerre polynomial of order $n$. For the lowest three values of $n$, we have $L_{0}\left(\eta^{2}\right)=1, L_{1}\left(\eta^{2}\right)=1-\eta^{2}$, and $L_{2}=1-2 \eta^{2}+\eta^{4} / 2$. This motion-dependent qubit rotation can be used to construct quantum logic gates $(51,52)$ 


\subsubsection{Spin-dependent optical forces}

An alternative method for coupling hyperfine qubits with the ion motion is to use laser beams to generate a dipole force that depends upon the state of the qubit $|S\rangle$ through atomic selection rules and appropriate polarization of the light. As an example, we consider the case of an optical coupling between $S_{1 / 2}$ and excited $P_{1 / 2}$ states, with a nuclear spin $I=1 / 2$. In this case, there are four ground states and four excited states, as depicted in Fig. 2. If the $S_{1 / 2}$ qubit states are $|\uparrow\rangle$ and $|\downarrow\rangle$ states, then a $\sigma^{+}$-polarized laser beam will couple the $|\uparrow\rangle$ to the excited state, with $|\downarrow\rangle$ decoupled.

Such a "spin-dependent force" can take many forms. For instance, the ion can be placed in an intensity gradient of a laser beams through focusing or through application of a standing-wave. ${ }^{(22)}$ Alternatively, off-resonant laser beams with a difference frequency near the trap frequency $\omega$ can be applied to the ion (a "walking wave" field), resulting in a resonant (qubit state-dependent) displacement of the motional state in phase space. $(53,56,57)$ Spin-dependent optical forces underly the "push" gate of Ref. 22 and the geometric phase gate discussed below ${ }^{(53)}$. Finally, we note the possibility of applying pairs of counterpropagating light pulses of duration $\tau \ll 1 / \gamma_{e}$, that resonantly drive transitions from one qubit state to the excited $P$ state and back down, accompanied by a $2 \hbar k$ impulse from the recoil of the absorption. ${ }^{(55)}$ This is the basis for the fast gate scheme proposed by Garcia-Rippol et al.. ${ }^{(54)}$

\subsubsection{Comparison of couplings}

The above two methods can be considered as formally equivalent, both involving a qubit state-dependent interaction with the ion coordinate that can be subsequently coupled to another ion through the Coulomb force. The original Cirac-Zoller coupling ${ }^{(1)}$ requires the preparation of the quantum state of ion motion to the $|n=0\rangle$ ground state, whereas most of the other couplings require preparation of the motion to within the LambDicke regime, where $\eta^{2}(\langle n\rangle+1) \ll 1$, where $\langle n\rangle$ is tha average number of (thermal) vibrational quanta in the ion motion.

HF qubit gates based on SRT couplings allow the creation of entangling quantum gates that change the qubit state, such as the CNOT gate, whereas spin-dependent optical forces generally provide gates that do not change the qubit state, such as the phase gate. Therefore, spin-dependent optical force gates can provide better isolation from errors associated with qubit rotations such as the residual coupling to spectator atomic levels. In particular, this imples that the gate speed for this method can be higher than that of SRT-based gates. ${ }^{(54)}$ However, spin-dependent optical forces 
appear to be applicable only to magnetically sensitive HF qubit states, thus the qubits in this case may be more susceptable to fluctuating magnetic fields. Moreover, HF qubit rotations must ultimately be applied in any case for universal quantum logic, so it is likely that both methods will be important in future work. As mentioned in the case of single qubit rotations based on stimulated Raman transitions, a common fundamental source of error in all coupling schemes is spontaneous emission from the excited state $|e\rangle$ (lifetime $1 / \gamma_{e}$ ) during the laser-induced coupling. We find that the probability of spontaneous emission per entangling gate is roughly

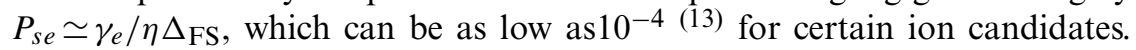

\section{GATE SCHEMES AND DEMONSTRATIONS}

The basic elements of the original Cirac-Zoller gate, ${ }^{(1)}$ a CNOT gate between the motion and the internal state of a single trapped ion was implemented in 1995.(45) The full Cirac-Zoller gate on two ions was implemented with about $70 \%$ fidelity in 2003. ${ }^{(38)}$ In 1999, Sørensen and Mølmer ${ }^{(46,47)}$ and also Solano et al. ${ }^{(48)}$ suggested an alternative gate scheme. Compared to the original Cirac and Zoller gate, ${ }^{(1)}$ the latter proposal has the advantages that (i) laser-beam focusing (for individual ion addressing) is not required, (ii) it can be carried out in one step, (iii) it does not require use of an additional internal state, and (iv) it does not require precise control of the motional state (as long as the Lamb-Dicke limit is satisfied). Based on this approach, the NIST group realized a universal gate between two spin qubits ${ }^{(49,50)}$ that was also used to demonstrate a particular four-qubit gate. ${ }^{(49)}$

A variation of the original Cirac-Zoller gate was demonstrated in 2002, relying on Raman carrier operations as discussed above. Here, the Lamb-Dicke parameter $\eta$ was set so that the carrier operations depended upon the motional state in a particular way ${ }^{(51,52)}$, resulting in a $\pi$-phase gate with a single pulse of light. Compared to the previously realized CNOT gate between motion and spin, ${ }^{(4)}$ this gate has the advantages that (i) it requires one step instead of three, (ii) it does not require an auxiliary internal state, and (iii) it is immune to Stark shifts caused by off-resonant sideband couplings. ${ }^{(4)}$

In 2003, the NIST group demonstrated a $\pi$-phase gate between two trapped ion qubits ${ }^{(53)}$ based on a qubit state-dependent force; with this gate a Bell state with $97 \%$ fidelity was generated. The gate realized the transformations: $|\downarrow\rangle|\downarrow\rangle \rightarrow|\downarrow\rangle|\downarrow\rangle,|\downarrow\rangle|\uparrow\rangle \rightarrow \mathrm{e}^{\mathrm{i} \pi / 2}|\downarrow\rangle|\uparrow\rangle,|\uparrow\rangle|\downarrow\rangle \rightarrow \mathrm{e}^{\mathrm{i} \pi / 2}|\uparrow\rangle|\downarrow\rangle$, and $|\uparrow\rangle|\uparrow\rangle \rightarrow|\uparrow\rangle|\uparrow\rangle$. Combined with single bit rotations, this operation can yield either a $\pi$-phase gate or the CNOT operation. 


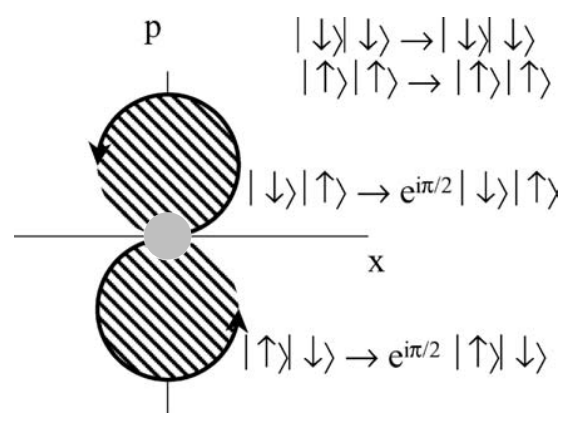

Fig. 4. Schematic representation of the displacements of the axial stretch-mode amplitude in phase space for the four basis states of the two spin qubits. The detuning and amplitude of the displacements are chosen to give a $\pi / 2$ phase shift on the $|\downarrow\rangle|\uparrow\rangle$ and $|\uparrow\rangle|\downarrow\rangle$ states while the $|\downarrow\rangle|\downarrow\rangle$ and $|\uparrow\rangle|\uparrow\rangle$ states are unaffected because the optical dipole forces for these states do not couple to the stretch mode.

The gate relies in part on properties of motional states as they are displaced in phase space. For a closed trajectory in phase space, the overall quantum state acquires an phase shift that depends on the area enclosed by the path. The second element required for the gate is to make the path area be spin-dependent. This is accomplished by making the displacement in phase space with a spin-dependent optical dipole force as was done in previous experiments. ${ }^{(56,57)}$

To implement this gate on two ions, the Raman transition beams were separated in frequency by $\sqrt{3} \omega_{z}+\delta$, where $\sqrt{3} \omega_{z}$ is the stretch-mode frequency for two ions and $\delta$ is a small detuning (below). The separation of the ions was adjusted to be an integer multiple of $2 \pi / \Delta k$ so that the optical-dipole force (from the "walking" standing wave) on each ion was in the same direction if the ions were in the same spin state but, due to the choice of laser polarizations, in opposite directions if the spin states were different. This had the effect that the application of the laser beams to the $|\downarrow\rangle|\uparrow\rangle$ and $|\uparrow\rangle|\downarrow\rangle$ states caused excitation on the stretch mode but the $\mid \downarrow$ \rangle$|\downarrow\rangle$ and $|\uparrow\rangle|\uparrow\rangle$ states were unaffected. The detuning $\delta$ and duration of the displacement pulses were chosen to make one complete (circular) path in phase space with an area that gave a phase shift of $\pi / 2$ on the $|\downarrow\rangle|\uparrow\rangle$ and $|\uparrow\rangle|\downarrow\rangle$ states. This is shown schematically in Fig. 4.

\section{CONCLUSION}

The trapped ion system is arguably one of the most attractive candidates for large-scale quantum computing. Here, we have concentrated 
on the use of atomic hyperfine ground states as qubits, the most stable quantum bit known. With a rich variety of schemes for generating entangling quantum logic gates between HF qubits based on externally applied laser fields, it appears that the scale up procedure is now limited by the fabrication of more complex trap arrays and the precise control of laser fields to produce high fidelity gates.

\section{REFERENCES}

1. J. I. Cirac and P. Zoller, Phys. Rev. Lett. 74, 4091 (1995).

2. D. P. DiVincenzo, in Scalable Quantum Computers, edited by S. L. Braunstein, H. K. Lo, and P. Kok (Wiley-VCH, Berlin, 2001), pp. 1-13.

3. A. Steane, Appl. Phys. B 64, 623 (1997).

4. D.J. Wineland, C.R. Monroe, W.M. Itano, D. Leibfried, B.E. King, and D.M. Meekhof, J. Res. Nat. Inst. Stand. Tech. 103, 259 (1998).

5. D.J. Wineland, C.R. Monroe, W.M. Itano, B.E. King, D. Leibfried, D.M. Meekhof, C.J. Myatt, and C.S. Wood, Fortschritte der Physik 46, 363 (1998).

6. D. F. V. James, in Scalable Quantum Computers, edited by S. L. Braunstein, H. K. Lo, and P. Kok (Wiley-VCH, Berlin, 2001), pp. 53-68.

7. G. J. Milburn, S. Schneider, and D. F. V. James, in Scalable Quantum Computers, edited by S. L. Braunstein, H. K. Lo, and P. Kok (Wiley-VCH, Berlin, 2001), pp. 31-40.

8. J. F. Poyatos, J. I. Cirac, and P. Zoller, in Scalable Quantum Computers, edited by S. L. Braunstein, H. K. Lo, and P. Kok (Wiley-VCH, Berlin, 2001), pp. 15-30.

9. A. Sørensen and K. Mølmer, in Scalable Quantum Computers, edited by S. L. Braunstein, H. K. Lo, and P. Kok (Wiley-VCH, Berlin, 2001), pp. 41-52.

10. A. M. Steane and D. M. Lucas, in Scalable Quantum Computers, edited by S. L. Braunstein, H. K. Lo, and P. Kok (Wiley-VCH, Berlin, 2001), pp. 69-88.

11. C. Monroe, Nature 416, 238 (2002).

12. D. J. Wineland, in Experimental Quantum Computation and Information, Proc. Int. School of Physics "Enrico Fermi", edited by F. D. Martini and C. Monroe (IOS Press, Amsterdam, 2002), Vol. 148, pp. 165-196.

13. D.J. Wineland, M. Barrett, J. Britton, J. Chiaverini, B.L. DeMarco, W.M. Itano, B.M. Jelenkovic, C. Langer, D. Leibfried, V. Meyer, T. Rosenband, and T. Schaetz, Phil. Trans. R. Soc. Lond. A 361, 1349 (2003).

14. S. Gulde, H. Häffner, M. Riebe, G. Lancaster, A. Mundt, A. Kreuter, C. Russo, C. Becher, J. Eschner, F. Schmidt-Kaler, I.L. Chuang, and R. Blatt, in Proceedings of the XVIII International Conference on Atomic Physics, edited by H. R. Sadeghpour, E. J. Heller, and D. E. Pritchard (World Scientific, Singapore, 2003), pp. 293-302.

15. D.J. Wineland, D. Leibfried, B.L. DeMarco, V. Meyer, M.A. Rowe, A. Ben Kish, M. Barrett, J. Britton, J. Hughes, W.M. Itano, B.M. Jelenkovic, C. Langer, D. Lucas and T. Rosenband, in Proceedings of the XVIII International Conference on Atomic Physics, edited by H. R. Sadeghpour, E. J. Heller, and D. E. Pritchard (World Scientific, Singapore, 2003), pp. 263-272.

16. C. Wunderlich and C. Balzer, quant-ph/0305129 (2003).

17. P. K. Ghosh, Ion Traps (Clarendon Press, Oxford, 1995).

18. C. A. Schrama, E. Peik, W. W. Smith, and H. Walther, Opt. Commun. 101, 32 (1993).

19. R. G. DeVoe, Phys. Rev. A 58, 910 (1998). 
20. H.C, Nägerl, W. Bechter, J, Eschner, F. Schmidt-Kaler, and R. Blatt, Appl. Phys. B 66, 603 (1998).

21. P. A. Barton, C. J. S. Donald, D. M. Lucas, D. A. Stevens, A. M. Steane, and D. N. Stacey, Phys. Rev. A 62, 032503 (2000).

22. J. I. Cirac and P. Zoller, Nature 404, 579 (2000).

23. G.R. Guthöhrlein, M. Keller, K. Hayasaka, W. Lange, H. Walther, Nature 414, 49 (2001).

24. L. Hornekær, N. Kjærgaard, A. M. Thommesen, and M. Drewsen, Phys. Rev. Lett. 86, 1994 (2001).

25. D. J. Berkeland, Rev. Sci. Instrum. 73, 2856 (2002).

26. D. Kielpinski, C. Monroe, and D. J. Wineland, Nature 417, 709 (2002).

27. M. Madsen, D. Stick, W. K. Hensinger, J. A. Rabchuk, and C. Monroe, quant-ph 0401047 (2004).

28. M.A. Rowe, A. Ben Kish, B.L. DeMarco, D. Leibfried, V. Meyer, J. Britton, J. Hughes, W.M. Itano, B.M. Jelenkovic, C. Langer, T. Rosenband, and D.J. Wineland, Quant. Inform. Comp. 2, 257 (2002).

29. A. Steane, C. F. Roos, D. Stevens, A. Mundt, D. Leibfried, F. Schmidt-Kaler, and R. Blatt, Phys. Rev. A 62, 042305 (2000).

30. J. I. Cirac, P. Zoller, H. J. Kimble, and H. Mabuchi, Phys. Rev. Lett. 78, 3221 (1997).

31. T. Pellizzari, Phys. Rev. Lett. 79, 5242 (1997).

32. L.-M. Duan, B. B. Blinov, D. L. Moehring, and C. Monroe, quant-ph 0401032 (2004).

33. H. Rohde, S. T. Gulde, C. F. Roos, P. A. Barton, D. Leibfried, J. Eschner, F. SchmidtKaler, R. Blatt, J. Opt. B: Quantum Semiclass. Opt. 3, S34 (2001).

34. B. B. Blinov, L. Deslauriers, P. Lee, M. J. Madsen, R. Miller, and C. Monroe, Phys. Rev. A 65, 040304 (2002).

35. M. Barrett, B.L. DeMarco, T. Schaetz, D. Leibfried, J. Britton, J. Chiaverini, W.M. Itano, B.M. Jelenkovic, J.D. Jost, C. Langer, T. Rosenband, and D.J. Wineland, Phys. Rev. A 68, 042302 (2003).

36. P. T. H. Fisk, Rep. Prog. Phys. 60, 761 (1997).

37. In Proceedings of the 6th Symposium on Frequency Standards and Metrology, edited by P. Gill (World Scientific, Singapore, 2002).

38. F. Schmidt-Kaler, H. Häffner, M. Riebe, S. Gulde, G. P. T. Lancaster, T. Deuschle, C. Becher, C. F. Roos, J. Eschner and R. Blatt, Nature 422, 408 (2003).

39. R. J. Rafac, B. C. Young, J. A. Beall, W. M. Itano, D. J. Wineland, and J. C. Bergquist, Phys. Rev. Lett. 85, 2462 (2000).

40. F. Schmidt-Kaler, S. Gulde, M. Riebe, T. Deuschle, A. Kreuter, G. Lancaster, C. Becher, J. Eschner, H. Häffner, and R. Blatt, J. Phys. B: At. Mol. Opt. Phys. 36, 623 (2003).

41. H. Häffner, S. Gulde, M. Riebe, G. Lancaster, C. Becher, J. Eschner, F. Schmidt-Kaler, and R. Blatt, Phys. Rev. Lett. 90, 143602 (2003).

42. R. Blatt and P. Zoller, Eur. J. Phys. 9, 250 (1988).

43. D.J. Wineland, J.J. Bollinger, W.M. Itano, F.L. Moore, and D.J. Heinzen, Phys. Rev. A 46, R6797 (1992).

44. F. Mintert and C. Wunderlich, Phys. Rev. Lett. 87, 257904 (2001).

45. C.R. Monroe, D.M. Meekhof, B.E. King, W.M. Itano, and D.J. Wineland, Phys. Rev. Lett. 75, 4714 (1995).

46. A. Sørensen and K. Mølmer, Phys. Rev. Lett. 82, 1971 (1999).

47. A. Sørensen and K. Mølmer, Phys. Rev. A 62, 02231 (2000).

48. E. Solano, R. L. de Matos Filho, and N. Zagury, Phys. Rev. A 59, 2539 (1999).

49. C.A. Sackett, D. Kielpinski, B.E. King, C. Langer, V. Meyer, C.J. Myatt, M.A. Rowe, Q.A. Turchette, W.M. Itano, D.J. Wineland, and C.R. Monroe, Nature 404, 256 (2000). 
50. D. Kielpinski, V. Meyer, M.A. Rowe, C.A. Sackett, W.M. Itano, C.R. Monroe, and D.J. Wineland, Science 291, 1013 (2001).

51. B. DeMarco, A. Ben-Kish, D. Leibfried, V. Meyer, M. Rowe, B.M. Jelenkovic, W.M. Itano, J. Britton, C. Langer, T. Rosenband, D.J. Wineland, quant-ph/0208180 (2002).

52. C.R. Monroe, D. Leibfried, B.E. King, D.M. Meekhof, W.M. Itano, and D.J. Wineland, Phys. Rev. A 55, R2489 (1997).

53. D. Leibfried, B.L. DeMarco, V. Meyer, D. Lucas, M. Barrett, J. Britton, W.M. Itano, B.M. Jelenkovic, C. Langer, T. Rosenband, and D.J. Wineland, Nature 422, 412 (2003).

54. J. J. Garcia-Ripoll, J. I. Cirac, and P. Zoller, Phys. Rev. Lett. 91, 157901 (2003).

55. J. F. Poyatos, J. I. Circa, R. Blatt, and P. Zoller, Phys. Rev. A 54, 1532 (1996).

56. C. Monroe, D. M. Meekhof, B. E. King, and D. J. Wineland, Science 272, 1131 (1996).

57. C.J. Myatt, B.E. King, Q.A. Turchette, C.A. Sackett, D. Kielpinski, W.M. Itano, C.R. Monroe, and D.J. Wineland, Nature 403, 269 (2000).

58. X. Wang, A. Sørensen, and K. Mølmer, Phys. Rev. Lett. 86, 3907 (2001). 